\title{
Effects of strain rates on kinetics of elements of repeated pattern structures: A continuous modeling approach
}

\author{
A. Salehian \\ Department of Mechanical and Mechatronics Engineering, University of Waterloo, Waterloo, Ontario, Canada \\ N2L $3 G 1$ \\ Tel.: +1 519888 4567,Ext. 38531; Fax: +1 519888 4333; E-mail: salehian@uwaterloo.ca
}

Received 5 July 2010

Revised 10 June 2011

Abstract. Space inflatable technology is a promising solution to placing large metrology systems in space. Lighter weight, higher packaging efficiency, and easier maintenance are among a few of their advantages over mechanically deployed structures. On the other hand, their large volume after deployment makes them susceptible to disturbances in space. Therefore, vibration control is one major issue for this technology. The present work is an extension of the previous work of the author on continuum modeling of these structures for their vibrations analysis. Kinetic and strain energy expressions of the fundamental lattice elements of a structure are expanded in terms of the nodal displacement components. Certain assumptions are made to reduce the order of strain components in a three-dimensional structure in order to find the equivalent continuum model. Additionally, this work includes the effects of strain rates on the kinetics of these structures. The frequency results for various structures are compared to those of a previous model which neglects such effects. It is shown that the frequency changes are noticeable when the strain rate components are included in the kinetic energy derivations.

Keywords: Homogenization, continuum modeling, lattice structures, strain rate, energy equivalence approach

\section{Nomenclature}

\begin{tabular}{|c|c|c|c|}
\hline$A_{L}, A_{d}, A_{b}$ & $\begin{array}{l}=\text { cross-sectional area of longerons, diago- } \\
\text { nals and battens }\end{array}$ & $U$ & $=$ strain energy \\
\hline$A^{(k)}$ & $=$ cross-sectional area of member $k$ & $u, v, w$ & $\begin{array}{l}=\text { displacement components at cross-section } \\
\text { in }(x, y, z) \text { directions }\end{array}$ \\
\hline$E_{L}, E_{d}, E_{b}$ & $\begin{array}{l}=\text { modulus of elasticity of longerons, diago- } \\
\text { nals and battens }\end{array}$ & $u_{0}, v_{0}, w_{0}$ & $\begin{array}{l}=\text { displacement components evaluated at the } \\
\text { center of the cross-section }\end{array}$ \\
\hline$E^{(k)}$ & $=$ modulus of elasticity of member $k$ & $V_{x, y, z}$ & $\begin{array}{l}=\text { Nodal velocity components of a bar } \\
\text { member }\end{array}$ \\
\hline$L_{L}, L_{d}, L_{b}$ & $=$ length of longerons, diagonals, battens & $x, y, z$ & $=$ Cartesian coordinates \\
\hline$L^{(k)}$ & $=$ length of member $k$ & $\phi_{x}, \phi_{y}, \phi_{z}$ & $\begin{array}{l}=\text { rotational degrees of freedom of the cross- } \\
\text { section }\end{array}$ \\
\hline$L_{t}$ & $=$ total length of the truss & $\begin{array}{l}\varepsilon_{x, 0}, \varepsilon_{y, 0}, \varepsilon_{z, 0} \\
\varepsilon_{x y, 0}, \varepsilon_{x z, 0}, \varepsilon_{y z, 0}\end{array}$ & $\begin{array}{l}=\text { strain components evaluated at } \\
\text { the center of the cross-section }\end{array}$ \\
\hline$l_{i}^{(k)}(i=1 \rightarrow 3)$ & $=$ directional cosine of member $\mathrm{k}$ & $\varepsilon_{i j}^{(k)}$ & $=$ strain components of member $\mathrm{k}$ \\
\hline$m^{(k)}$ & $=$ mass of member $\mathrm{k}$ & $\kappa_{y, 0}, \kappa_{z, 0}, \kappa_{x, 0}$ & $\begin{array}{l}=\text { curvatures evaluated at the center of the } \\
\text { cross-section }\end{array}$ \\
\hline$T$ & $=$ kinetic energy & $\rho_{L}, \rho_{d}, \rho_{b}$ & $=$ density of longerons, diagonals and battens \\
\hline$t$ & $=$ time & $\omega$ & $=$ natural frequency $(\mathrm{rad} / \mathrm{sec})$ \\
\hline
\end{tabular}




\section{Introduction}

Recently, there has been increasing demands for placing large metrology systems in the Medium Earth Orbit (MEO) due to their improved coverage. A satellite system operating in MEO has to be so large that they cannot be launched on existing rockets (Chmielewski [1]). Inflatable satellites that can be compressed into small packages upon their launch are viable solutions for placing large antennas in orbits. These structures usually comprise of lattice elements due to their large stiffness to mass ratios. As these structures are too large to be ground tested, there is primary need for understanding their underlying dynamic behavior. Finite Element Analysis (FEA) requires a significant amount of storage capacity to obtain reliable solutions and hence not the most suitable for control applications. Alternatively, continuum modeling techniques are effective methods for modeling large structures of repeated patterns. In particular, they can effectively be used with developed control techniques for distributed parameter systems. A complete survey of the continuum modeling approaches and their advantages are listed in $[2-4]$.

The continuum modeling techniques developed in the literature can be categorized in to two major categories. One contains some ad-hoc schemes that are found by relating the force and deformation of a small segment of a lattice structure with those of its equivalent continuum model [5-10]. The second category results from a more accurate approach which employs an energy equivalence concept [2,11-18]. In all the previous work on continuum modeling which employs the concept of the energy equivalence, the effects of the local strain rate components are ignored in the kinetic energy derivations for the fundamental elements. The present work is aimed to fill this gap. Including these strain rates in the present work results in significantly different partial differential equations for these structures. The numerical results for natural frequencies are compared to a previous model which ignores these effects and leads to overestimating the frequencies.

\section{Theory}

The first step in finding an equivalent continuum model is to get the strain and kinetic energy expressions for the fundamental lattice element. Since the structure has a repeated pattern, these expressions can be used to derive the governing partial differential equations for an equivalent one-dimensional model provided that certain assumptions are made to relate the strain components from a three-dimensional domain to a one-dimensional domain [2,11-14]. The proceeding sections present such derivations. The major difference between the present approach and the previously established methods is in the inclusion of the local strain and strain rate components in the kinetic energy derivations.

\subsection{Strain energy derivations}

Similar to the previous work of the authors, the strain components for the structure can be found using the relevant assumptions for the displacement field of the element. Assuming a linearly varying displacement field at the cross section of the element we get:

$$
\begin{aligned}
& u(x, y, z)=u_{0}-y \phi_{z}+z \phi_{y} \\
& v(x, y, z)=v_{0}+y \varepsilon_{y, 0}+z\left[-\phi_{x}+\varepsilon_{y z, 0}\right] \\
& w(x, y, z)=w_{0}+y\left[\phi_{x}+\varepsilon_{y z, 0}\right]+z \varepsilon_{z, 0}
\end{aligned}
$$

Having the displacement field, the strain components can be found using the spatial derivatives in each of the $x, y$ and $z$ directions. The derivation for these strain components of each of the members in a lattice denoted by ' $k$ ' can be found in the Appendix. Note that the strain components shown belong to a three-dimensional element and some key assumptions must be made before an equivalent one-dimensional model can be found. Some of these assumptions as used previously by authors [2,11-14] are as follow:

The local free deformation forces associated with the local deformations should vanish to find the equivalent one-dimensional model. 


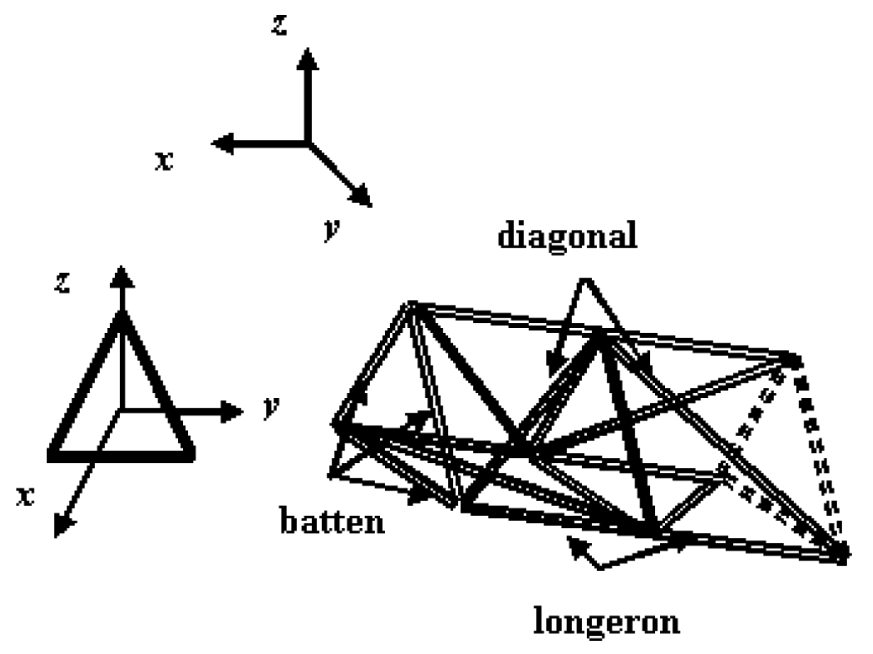

Fig. 1. Schematic of the fundamental element of the inflatable lattice structure.

$$
\begin{aligned}
\frac{\partial U}{\partial\left[\frac{\partial \varepsilon_{x, 0}}{\partial x}\right]} & =\frac{\partial U}{\partial\left[\frac{\partial \kappa_{y, 0}}{\partial x}\right]}=\frac{\partial U}{\partial\left[\frac{\partial \kappa_{z, 0}}{\partial x}\right]}=\frac{\partial U}{\partial\left[\frac{\partial \varepsilon_{x y, 0}}{\partial x}\right]}=\frac{\partial U}{\partial\left[\frac{\partial \varepsilon_{x z, 0}}{\partial x}\right]} \\
& =\frac{\partial U}{\partial\left[\frac{\partial \kappa_{x, 0}}{\partial x}\right]}=\frac{\partial U}{\partial\left[\frac{\partial^{2} \varepsilon_{y, 0}}{\partial x^{2}}\right]}=\frac{\partial U}{\partial\left[\frac{\partial^{2} \varepsilon_{z, 0}}{\partial x^{2}}\right]}=\frac{\partial U}{\partial\left[\frac{\partial^{2} \varepsilon_{y z, 0}}{\partial x^{2}}\right]}=0
\end{aligned}
$$

The assumption of a shear deformation type structure results in that the shear stress in the plane of the cross section and the extensional stress components along the $y$ and $z$ directions should vanish.

$$
\frac{\partial U}{\partial \varepsilon_{y, 0}}=\frac{\partial U}{\partial \varepsilon_{z, 0}}=\frac{\partial U}{\partial \varepsilon_{y z, 0}}=0
$$

The last assumption is the compatibility condition which forces the strain components at the cross section to be identical for two adjacent elements. This means that they should have zero local variations with respect to $x$, therefore,

$$
\frac{\partial \varepsilon_{y, 0}}{\partial x}=\frac{\partial \varepsilon_{z, 0}}{\partial x}=\frac{\partial \varepsilon_{y z, 0}}{\partial x}=0
$$

The above 15 Eqs (2)-(4) will result in the solution for 15 unknown strain components. There are a total of 21 strain components and their derivatives for the fundamental lattice element as shown in the Appendix. This results in a total of 6 independent strain components which are similar to those of a one-dimensional lattice. These are $\varepsilon_{x, 0}, \kappa_{y, 0}, \kappa_{z, 0}, \varepsilon_{x y, 0}, \varepsilon_{x z, 0}$, and $\kappa_{x, 0}$. Since these strain components depend on $x$ only (single coordinate), the strain energy is similar to that of a one-dimensional structure. The strain energy of the fundamental element can be found as:

$$
\begin{aligned}
U & =3 A_{L} E_{L} L_{L} \varepsilon_{x, 0}^{2}+\frac{1}{2} A_{L} E_{L} L_{b}^{2} L_{L} \kappa_{y, 0}^{2}+\frac{1}{2} A_{L} E_{L} L_{b}^{2} L_{L} \kappa_{z, 0}^{2}+\frac{24 A_{d} A_{L} E_{d} E_{L} L_{b}^{2} L_{d} L_{L}^{2}}{A_{d} E_{d} L_{d} L_{L}^{3}+4 A_{L} E_{L}\left(L_{b}^{2}+L_{L}^{2}\right)^{2}} \varepsilon_{x y, 0}^{2} \\
& +\frac{24 A_{d} A_{L} E_{d} E_{L} L_{b}^{2} L_{d} L_{L}^{2}}{A_{d} E_{d} L_{d} L_{L}^{3}+4 A_{L} E_{L}\left(L_{b}^{2}+L_{L}^{2}\right)^{2}} \varepsilon_{x z, 0}^{2}+\frac{A_{d} A_{L} E_{d} E_{L} L_{b}^{4} L_{d} L_{L}^{2}}{4\left(A_{d} E_{d} L_{d} L_{L}^{3}+A_{L} E_{L}\left(L_{b}^{2}+L_{L}^{2}\right)^{2}\right)} \kappa_{x, 0}^{2}
\end{aligned}
$$

\subsection{Kinetic energy derivations}

The next step in finding the continuum model is to find the kinetic energy expressions for the fundamental lattice element. In all the previous work [2,11-14] the effects of the strain rate and the strain components across the length 
of the element were ignored in the kinetic energy derivations. This is equivalent to making the assumption that the three bending coordinates $(u, v, w)$ are the same along the length of the fundamental element. The present work is focused on including these strain components and their effects on the overall dynamics of the structure. To find the kinetic energy of the fundamental element, the kinetic energy of each of the bar members in the element as shown in Fig. 1 must be found. It can be shown that the kinetic energy for a bar member can be written as:

$$
T=\frac{m}{6}\left(V_{1 x}^{2}+V_{1 y}^{2}+V_{1 z}^{2}+V_{2 x}^{2}+V_{2 y}^{2}+V_{2 z}^{2}+V_{1 x} V_{2 x}+V_{1 y} V_{2 y}+V_{1 z} V_{2 z}\right)
$$

where $m$ denotes the mass of the bar, $V$ is the nodal velocity, first subscript denotes each of the two end nodes of a bar member and the second subscript denotes the velocity components. Also, using the shear strain can be written in the form of:

$$
\begin{aligned}
& \varepsilon_{x y, 0}=0.5\left(\frac{\partial v}{\partial x}-\phi_{z, 0}\right) \Rightarrow \frac{\partial v}{\partial x}=2 \varepsilon_{x y, 0}+\phi_{z, 0} \\
& \varepsilon_{x z, 0}=0.5\left(\frac{\partial w}{\partial x}+\phi_{y, 0}\right) \Rightarrow \frac{\partial w}{\partial x}=2 \varepsilon_{x z, 0}-\phi_{y, 0}
\end{aligned}
$$

Using the time derivative of the displacement components in Eq. (11), Eq. (6) and the strains in Eq. (7), we get the following for the kinetic energy of the element:

$$
\begin{aligned}
T & =\frac{m^{(k)}}{6}\left(\left(\frac{\partial}{\partial t}\left(u_{0}+x_{1}^{(k)} \varepsilon_{x, 0}-y_{1}^{(k)} \phi_{z, 0}+z_{1}^{(k)} \phi_{y, 0}\right)\right)^{2}+\left(\frac{\partial}{\partial t}\left(v_{0}+x_{1}^{(k)}\left(2 \varepsilon_{x y, 0}+\phi_{z, 0}\right)-z_{1}^{(k)} \phi_{x, 0}\right)\right)^{2}\right. \\
& +\left(\frac{\partial}{\partial t}\left(w_{0}+x_{1}^{(k)}\left(2 \varepsilon_{x z, 0}-\phi_{y, 0}\right)+y_{1}^{(k)} \phi_{x, 0}\right)\right)^{2}+\left(\frac{\partial}{\partial t}\left(u_{0}+x_{2}^{(k)} \varepsilon_{x, 0}-y_{2}^{(k)} \phi_{z, 0}+z_{2}^{(k)} \phi_{y, 0}\right)\right)^{2} \\
& +\left(\frac{\partial}{\partial t}\left(v_{0}+x_{2}^{(k)}\left(2 \varepsilon_{x y, 0}+\phi_{z, 0}\right)-z_{2}^{(k)} \phi_{x, 0}\right)\right)^{2}+\left(\frac{\partial}{\partial t}\left(w_{0}+x_{2}^{(k)}\left(2 \varepsilon_{x z, 0}-\phi_{y, 0}\right)+y_{2}^{(k)} \phi_{x, 0}\right)\right)^{2} \\
& +\frac{\partial}{\partial t}\left(u_{0}+x_{1}^{(k)} \varepsilon_{x, 0}-y_{1}^{(k)} \phi_{z, 0}+z_{1}^{(k)} \phi_{y, 0}\right) \frac{\partial}{\partial t}\left(u_{0}+x_{2}^{(k)} \varepsilon_{x, 0}-y_{2}^{(k)} \phi_{z, 0}+z_{2}^{(k)} \phi_{y, 0}\right) \\
& +\frac{\partial}{\partial t}\left(v_{0}+x_{1}^{(k)}\left(2 \varepsilon_{x y, 0}+\phi_{z, 0}\right)-z_{1}^{(k)} \phi_{x, 0}\right) \frac{\partial}{\partial t}\left(v_{0}+x_{2}^{(k)}\left(2 \varepsilon_{x y, 0}+\phi_{z, 0}\right)-z_{2}^{(k)} \phi_{x, 0}\right) \\
& \left.+\frac{\partial}{\partial t}\left(w_{0}+x_{1}^{(k)}\left(2 \varepsilon_{x z, 0}-\phi_{y, 0}\right)+y_{1}^{(k)} \phi_{x, 0}\right) \frac{\partial}{\partial t}\left(w_{0}+x_{2}^{(k)}\left(2 \varepsilon_{x z, 0}-\phi_{y, 0}\right)+y_{2}^{(k)} \phi_{x, 0}\right)\right)
\end{aligned}
$$

The difference between the above kinetic energy and those of the previous work by the author in $[2,11]$ is:

$$
\begin{aligned}
\Delta T & =\frac{1}{2} L_{L}\left(-L_{L}\left(\left(\frac{\partial \varepsilon_{x, 0}}{\partial t}\right)^{2}+4\left(\left(\frac{\partial \varepsilon_{x y, 0}}{\partial t}\right)^{2}+\left(\frac{\partial \varepsilon_{x z, 0}}{\partial t}\right)^{2}\right)\right)\left(3 A_{b} L_{b} \rho_{b}+2 A_{d} L_{d} \rho_{d}+2 A_{L} L_{L} \rho_{L}\right)\right. \\
& -6 A_{b} L_{b} \rho_{b} \frac{\partial \varepsilon_{x, 0}}{\partial t} \frac{\partial u_{x, 0}}{\partial t}-12 A_{b} L_{b} \rho_{b} \frac{\partial \varepsilon_{x y, 0}}{\partial t} \frac{\partial v_{0}}{\partial t}+\left(12 A_{b} L_{b} L_{L} \rho_{b}+8 A_{L} L_{L}^{2} \rho_{L}\right) \frac{\partial \varepsilon_{x z, 0}}{\partial t} \frac{\partial \phi_{y, 0}}{\partial t} \\
& -\left(3 A_{b} L_{b} L_{L} \rho_{b}+2 A_{L} L_{L}^{2} \rho_{L}\right)\left(\frac{\partial \phi_{y, 0}}{\partial t}\right)^{2}+6 A_{b} L_{b} \rho_{b} \frac{\partial w_{0}}{\partial t}\left(-2 \frac{\partial \varepsilon_{x z, 0}}{\partial t}+\frac{\partial \phi_{y, 0}}{\partial t}\right) \\
& -12 A_{b} L_{b} L_{L} \rho_{b} \frac{\partial \varepsilon_{x y, 0}}{\partial t} \frac{\partial \phi_{z, 0}}{\partial t}-8 A_{L} L_{L}^{2} \rho_{L} \frac{\partial \varepsilon_{x y, 0}}{\partial t} \frac{\partial \phi_{z, 0}}{\partial t}-6 A_{b} L_{b} \rho_{b} \frac{\partial v_{0}}{\partial t} \frac{\partial \phi_{z, 0}}{\partial t} \\
& -3 A_{b} L_{b} L_{L} \rho_{b}\left(\frac{\partial \phi_{z, 0}}{\partial t}\right)^{2}-2 A_{L} L_{L}^{2} \rho_{L}\left(\frac{\partial \phi_{z, 0}}{\partial t}\right)^{2} \\
& \left.+2 A_{d} L_{L} L_{d} \rho_{d}\left(\left(4 \frac{\partial \varepsilon_{x z, 0}}{\partial t}-\frac{\partial \phi_{y, 0}}{\partial t}\right) \frac{\partial \phi_{y, 0}}{\partial t}-\frac{\partial \phi_{z, 0}}{\partial t}\left(4 \frac{\partial \varepsilon_{x y, 0}}{\partial t}-\frac{\partial \phi_{z, 0}}{\partial t}\right)\right)\right)
\end{aligned}
$$




\subsection{Equations of motion}

Having the kinetic and strain energy of the fundamental element and considering that the structure consists of a repeating pattern of these elements, partial differential equations of motion can be found using Hamilton's principle. Since there are six strain components which can be expanded in terms of six displacement components, there will also be six coupled equations of motion pertaining to those coordinates:

$$
\begin{aligned}
& \left(-2 A_{b} L_{b} \rho_{b}-2 A_{d} L_{d} \rho_{d}-4 A_{L} L_{L} \rho_{L}\right) \frac{\partial^{2} \phi_{x}}{\partial t^{2}}+\frac{A_{d} A_{L} E_{d} E_{L} L_{b}^{2} L_{d} L_{L}^{2}}{A_{d} E_{d} L_{d} L_{L}^{3}+A_{L} E_{L} L_{d}^{2}} \frac{\partial^{2} \phi_{x}}{\partial x^{2}}=0 \\
& -6\left(A_{b} L_{b} \rho_{b}+A_{d} L_{d} \rho_{d}+A_{L} L_{L} \rho_{L}\right) \frac{\partial^{2} u}{\partial t^{2}}+\underbrace{L_{L}^{2}\left(3 A_{b} L_{b} \rho_{b}+2 A_{d} L_{d} \rho_{d}+2 A_{L} L_{L} \rho_{L}\right) \frac{\partial^{4} u}{\partial t^{2} \partial x^{2}}}_{\text {newterms }} \\
& +6 A_{L} E_{L} L_{L} \frac{\partial^{2} u}{\partial x^{2}}=0 \\
& -\left(A_{b} L_{b} \rho_{b}+A_{d} L_{d} \rho_{d}+2 A_{L} L_{L} \rho_{L}\right) \frac{\partial \phi_{z}}{\partial t^{2}} \\
& -A_{L} E_{L} L_{L}\left(\frac{24 A_{d} E_{d} L_{d} L_{L}}{A_{d} E_{d} L_{d} L_{L}^{3}+4 A_{L} E_{L}\left(L_{b}^{2}+L_{L}^{2}\right)^{2}}\left(\phi_{z}-\frac{\partial v}{\partial x}\right)-2 \frac{\partial \phi_{z}}{\partial x^{2}}\right)=0 \\
& -\left(3 A_{b} L_{b} \rho_{b}+3 A_{d} L_{d} \rho_{d}+3 A_{L} L_{L} \rho_{L}\right) \frac{\partial^{2} v}{\partial t^{2}}-\frac{6 A_{d} A_{L} E_{d} E_{L} L_{b}^{2} L_{d} L_{L}^{2}}{A_{d} E_{d} L_{d} L_{L}^{3}+4 A_{L} E_{L} L_{d}^{4}}\left(\frac{\partial \phi_{z}}{\partial x}-\frac{\partial^{2} v}{\partial x^{2}}\right) \\
& +\underbrace{L_{L}^{2}\left(1.5 A_{b} L_{b} \rho_{b}+A_{d} L_{d} \rho_{d}+A_{L} L_{L} \rho_{L}\right) \frac{\partial^{4} v}{\partial t^{2} \partial x^{2}}}_{\text {new terms }}=0 \\
& -\left(A_{b} L_{b} \rho_{b}+A_{d} L_{d} \rho_{d}+2 A_{L} L_{L} \rho_{L}\right) \frac{\partial^{2} \phi_{y}}{\partial t^{2}} \\
& -A_{L} E_{L} L_{L}\left(\frac{24 A_{d} E_{d} L_{d} L_{L}}{A_{d} E_{d} L_{d} L_{L}^{3}+4 A_{L} E_{L}\left(L_{b}^{2}+L_{L}^{2}\right)^{2}}\left(\phi_{y}+\frac{\partial w}{\partial x}\right)-2 \frac{\partial \phi_{y}}{\partial x^{2}}\right)=0 \\
& -\left(3 A_{b} L_{b} \rho_{b}+3 A_{d} L_{d} \rho_{d}+3 A_{L} L_{L} \rho_{L}\right) \frac{\partial^{2} w}{\partial t^{2}}+\frac{6 A_{d} A_{L} E_{d} E_{L} L_{b}^{2} L_{d} L_{L}^{2}}{A_{d} E_{d} L_{d} L_{L}^{3}+4 A_{L} E_{L} L_{d}^{4}}\left(\frac{\partial^{2} w}{\partial x^{2}}+\frac{\partial \phi_{y}}{\partial x}\right) \\
& +\underbrace{L_{L}^{2}\left(1.5 A_{b} L_{b} \rho_{b}+A_{d} L_{d} \rho_{d}+A_{L} L_{L} \rho_{L}\right) \frac{\partial^{4} w}{\partial t^{2} \partial x^{2}}}_{\text {new terms }}=0
\end{aligned}
$$

As it is shown each of the bending coordinates are coupled with the two rotations of the cross section components similar to those of the previous work of the author [11]. The difference between these equations and those of the previous work is in the terms $\frac{\partial^{4} v}{\partial t^{2} \partial x^{2}}$ and $\frac{\partial^{4} w}{\partial t^{2} \partial x^{2}}$ (curvature acceleration components). These terms are the results of accounting for the strain rates that were previously ignored in the kinetic energy derivations. As a result, the longitudinal Eq. (10-b) is also different due to the addition of the term $\frac{\partial^{4} u}{\partial t^{2} \partial x^{2}}$. The equation for twist Eq. (10-a) remains identical to that of the previous work of the author.

Finally, a simpler form of these equations can be found by making the assumptions for a non-shearable structure as well as ignoring the rotary inertia effects which is similar to the assumptions for an Euler-Bernoulli beam theory. The assumptions for the non-shearable model result in: 


$$
\begin{aligned}
& 2 \varepsilon_{x y, 0}=\left(\frac{\partial v}{\partial x}-\phi_{z, 0}\right)=0 \\
& 2 \varepsilon_{x z, 0}=\left(\frac{\partial w}{\partial x}+\phi_{y, 0}\right)=0
\end{aligned}
$$

Therefore, the kinetic energy can be found as:

$$
\begin{aligned}
T & =\frac{m^{(k)}}{6}\left(\left(\frac{\partial u_{0}}{\partial t}+x_{1}^{(k)} \frac{\varepsilon_{x, 0}}{\partial t}\right)^{2}+\left(\frac{\partial v_{0}}{\partial t}+2 x_{1}^{(k)} \frac{\partial \varepsilon_{x y, 0}}{\partial t}-z_{1}^{(k)} \frac{\partial \phi_{x, 0}}{\partial t}\right)^{2}+\left(\frac{\partial w_{0}}{\partial t}+2 x_{1}^{(k)} \frac{\partial \varepsilon_{x z, 0}}{\partial t}+y_{1}^{(k)} \frac{\partial \phi_{x, 0}}{\partial t}\right)^{2}\right. \\
& +\left(\frac{\partial u_{0}}{\partial t}+x_{2}^{(k)} \frac{\partial \varepsilon_{x, 0}}{\partial t}\right)^{2}+\left(\frac{\partial v_{0}}{\partial t}+2 x_{2}^{(k)} \frac{\partial \varepsilon_{x y, 0}}{\partial t}-z_{2}^{(k)} \frac{\partial \phi_{x, 0}}{\partial t}\right)^{2}+\left(\frac{\partial w_{0}}{\partial t}+2 x_{2}^{(k)} \frac{\partial \varepsilon_{x z, 0}}{\partial t}+y_{2}^{(k)} \frac{\partial \phi_{x, 0}}{\partial t}\right)^{2} \\
& +\left(\frac{\partial v_{0}}{\partial t}+2 x_{1}^{(k)} \frac{\partial \varepsilon_{x y, 0}}{\partial t}-z_{1}^{(k)} \frac{\partial \phi_{x, 0}}{\partial t}\right)\left(\frac{\partial v_{0}}{\partial t}+2 x_{2}^{(k)} \frac{\partial \varepsilon_{x y, 0}}{\partial t}-z_{2}^{(k)} \frac{\partial \phi_{x, 0}}{\partial t}\right) \\
& +\left(\frac{\partial w_{0}}{\partial t}+2 x_{1}^{(k)} \frac{\partial \varepsilon_{x z, 0}}{\partial t}+y_{1}^{(k)} \frac{\partial \phi_{x, 0}}{\partial t}\right)\left(\frac{\partial w_{0}}{\partial t}+2 x_{2}^{(k)} \frac{\partial \varepsilon_{x z, 0}}{\partial t}+y_{2}^{(k)} \frac{\partial \phi_{x, 0}}{\partial t}\right) \\
& \left.+\left(\frac{\partial u_{0}}{\partial t}+x_{1}^{(k)} \frac{\partial \varepsilon_{x, 0}}{\partial t}\right)\left(\frac{\partial u_{0}}{\partial t}+x_{2}^{(k)} \frac{\partial \varepsilon_{x, 0}}{\partial t}\right)\right)
\end{aligned}
$$

Using the kinetic and strain energy terms for the non-shearable model and Hamilton's principle, the equations of motion can be found as:

$$
\begin{aligned}
& \left(-2 A_{b} L_{b} \rho_{b}-2 A_{d} L_{d} \rho_{d}-4 A_{L} L_{L} \rho_{L}\right) \frac{\partial^{2} \phi_{x}}{\partial t^{2}}+\frac{A_{d} A_{L} E_{d} E_{L} L_{b}^{2} L_{d} L_{L}^{2}}{A_{d} E_{d} L_{d} L_{L}^{3}+A_{L} E_{L} L_{d}^{2}} \frac{\partial^{2} \phi_{x}}{\partial x^{2}}=0 \\
& -6\left(A_{b} L_{b} \rho_{b}+A_{d} L_{d} \rho_{d}+A_{L} L_{L} \rho_{L}\right) \frac{\partial^{2} u}{\partial t^{2}}+L_{L}^{2}\left(3 A_{b} L_{b} \rho_{b}+2 A_{d} L_{d} \rho_{d}+2 A_{L} L_{L} \rho_{L}\right) \frac{\partial^{4} u}{\partial t^{2} \partial x^{2}} \\
& +6 A_{L} E_{L} L_{L} \frac{\partial^{2} u}{\partial x^{2}}=0 \\
& -6\left(A_{b} L_{b} \rho_{b}+A_{d} L_{d} \rho_{d}+A_{L} L_{L} \rho_{L}\right) \frac{\partial^{2} v}{\partial t^{2}}+L_{L}^{2}\left(3 A_{b} L_{b} \rho_{b}+2 A_{d} L_{d} \rho_{d}+2 A_{L} L_{L} \rho_{L}\right) \frac{\partial^{4} v}{\partial t^{2} \partial x^{2}} \\
& -A_{L} E_{L} L_{b}^{2} L_{L} \frac{\partial^{4} v}{\partial x^{4}}=0 \\
& -6\left(A_{b} L_{b} \rho_{b}+A_{d} L_{d} \rho_{d}+A_{L} L_{L} \rho_{L}\right) \frac{\partial^{2} w}{\partial t^{2}}+L_{L}^{2}\left(3 A_{b} L_{b} \rho_{b}+2 A_{d} L_{d} \rho_{d}+2 A_{L} L_{L} \rho_{L}\right) \frac{\partial^{4} w}{\partial t^{2} \partial x^{2}} \\
& -A_{L} E_{L} L_{b}^{2} L_{L} \frac{\partial^{4} w}{\partial x^{4}}=0
\end{aligned}
$$

Note, that there are four equations of motion for the four coordinates of vibration. This is because the two rotations of the cross section in a non-shearable model are not independent coordinates as they are slopes of the bending coordinates. The equations for the longitudinal and twist coordinates Eq. (13-a) and (b) remain identical to those of shearable model Eq. (10-a) and (b).

\section{Numerical results}

The natural frequency results for the developed model are presented and compared with the results of the previous work of the author and the FEA. The material and geometrical properties of the inflatable structure which is based 
Table 1

Properties of the lattice

\begin{tabular}{|c|c|}
\hline Property & Value \\
\hline Length of longerons and battens & $3 m$ \\
\hline Area of longerons & $2.4322 \times 10^{-5} \mathrm{~m}^{2}$ \\
\hline Area of battens and diagonals & $4.0537 \times 10^{-4} \mathrm{~m}^{2}$ \\
\hline Modulus of longerons, battens and diagonals & $6.8948 \times 10^{10} \mathrm{~N} / \mathrm{m}^{2}$ \\
\hline Density of longerons, battens and diagonals & $1.7992 \times 10^{3} \mathrm{Kg} / \mathrm{m}^{3}$ \\
\hline
\end{tabular}

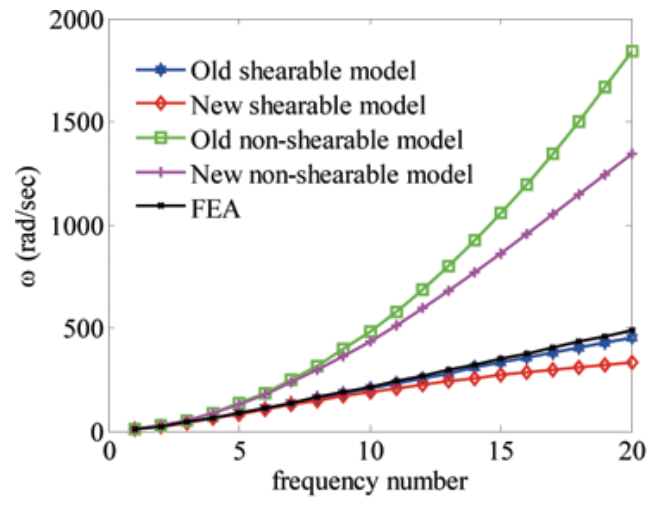

(a)

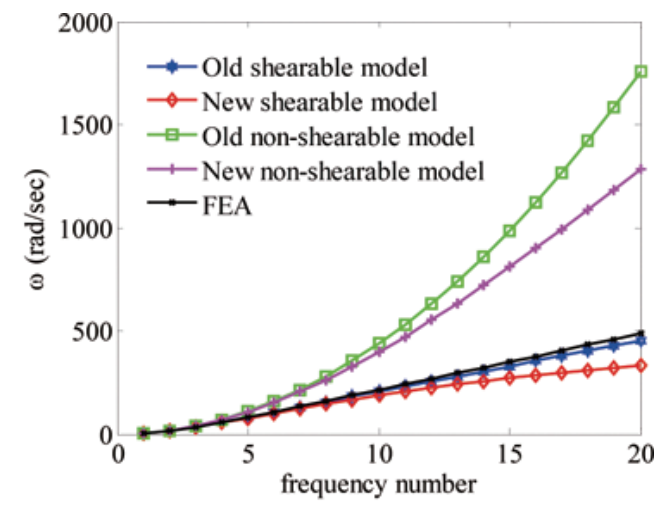

(b)

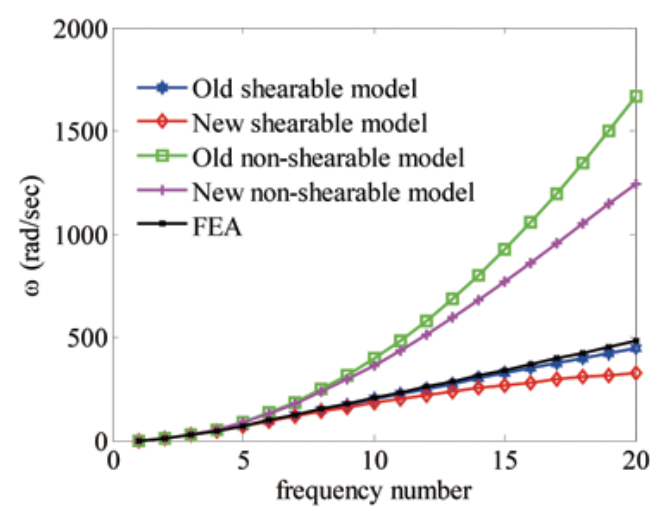

(c)

Fig. 2. Bending natural frequencies for a $120 \mathrm{~m}$ lattice structure for a) clamped-clamped; b) simply supported; c) free-free boundary condition.

on a government referenced model are presented in Table 1. Additionally, the schematic of the fundamental element for the inflatable antenna truss is shown in Fig. 1. The frequency results are found for various structure lengths and boundary conditions.

The first part of the results pertains to a comparison between the shearable and non-shearable models. As it is shown, the shearable model has better agreement with the FEA solution when compared to the non-shearable model. This is due to the fact that the rotary inertia and the shear strain effects, which are neglected by the non-shearable model, are taken into account in this model. Generally, ignoring the shear strain effects is equivalent to making the assumption of an infinite shear modulus for the structure which is essentially over-estimating the stiffness. This results in over-estimating the natural frequencies. Additionally, ignoring the rotary inertia effects in the kinetic energy results in under-estimating the kinetic energy which also results in over-estimating the natural frequencies. Therefore, the non-shearable model for which both of these effects are ignored has higher frequency estimations when compared to both shearable model and the FEA.

The second part of the results compare the new shearable model developed in this paper with the previous model 


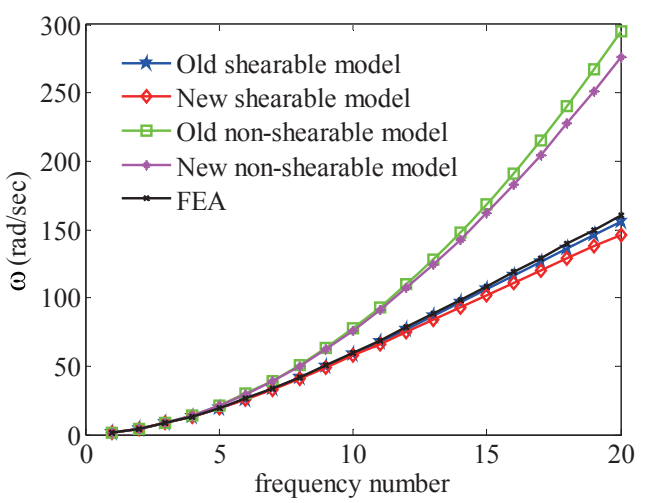

(a)

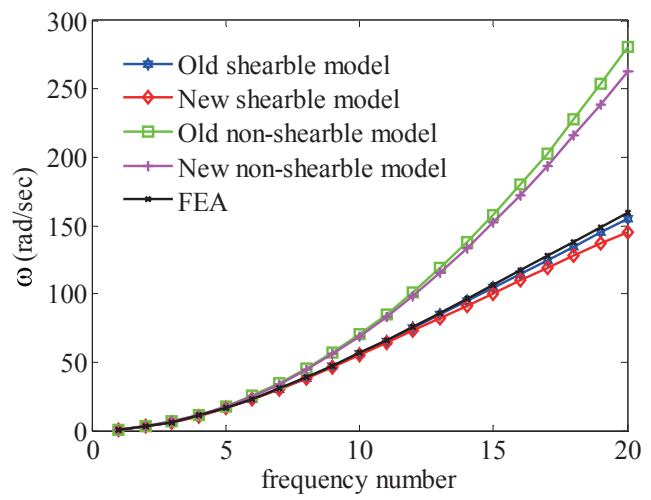

(b)

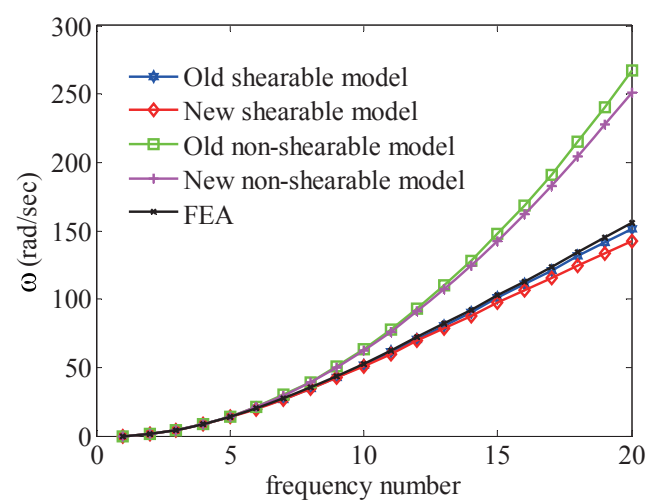

(c)

Fig. 3. Bending natural frequencies for a $300 \mathrm{~m}$ lattice structure for a) clamped-clamped b) simply supported c) free-free boundary condition.

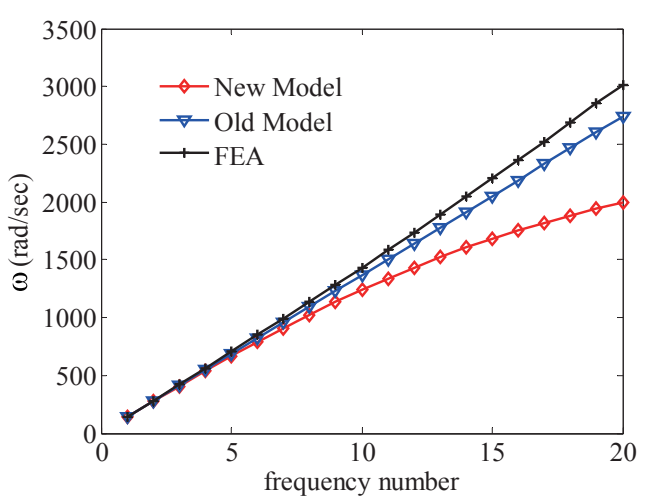

(a)

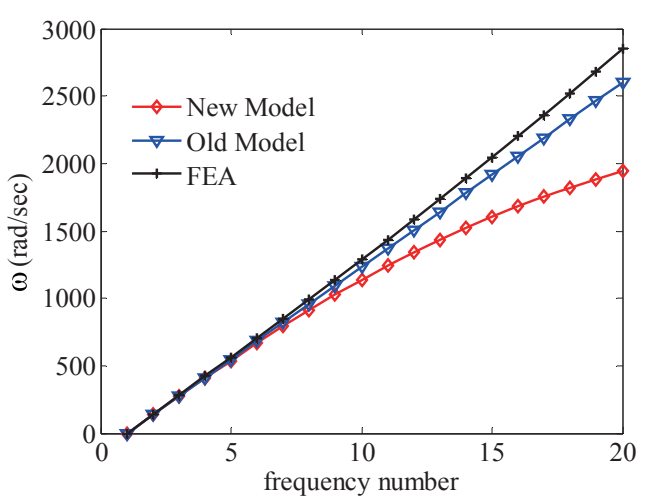

(b)

Fig. 4. Longitudinal natural frequencies for a $120 \mathrm{~m}$ lattice structure for a) clamped-clamped b) free-free boundary condition.

in which the strain rate effects are ignored in the kinetic energy derivations. As it is shown in Figs 2 and 3, the natural frequencies from the new shearable model show a noticeable change when compared to the previous model. Also, it can be seen that the FEA over-estimates the natural frequencies; this is expected as FEA solutions always find the upper bound of a Rayleigh Quotient problem [2,11].

Finally, the results for the longitudinal frequencies are presented in Figs 4 and 5. The results for these modes also reflect the changes in frequencies when compared to the previous shearable model which ignores these effects. 


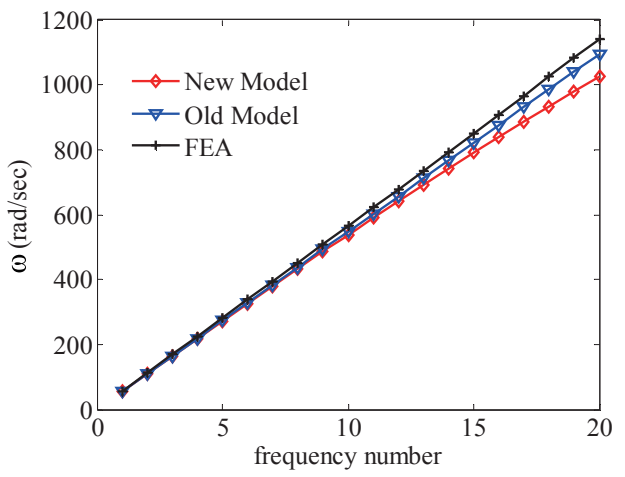

(a)

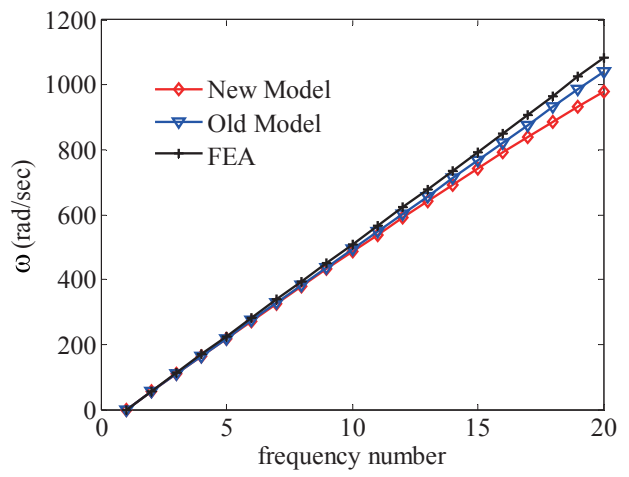

(b)

Fig. 5. Longitudinal natural frequencies for a $300 \mathrm{~m}$ lattice structure for a) clamped-clamped b) free-free boundary condition.

\section{Conclusion}

A homogenization technique which includes the strain rate components in the kinetics of structures with repeated pattern is developed. Numerical results for natural frequencies of various structures demonstrate a noticeable change when compared to those of a previous model which ignores the strain rate effects. It is shown that these changes are more significant for higher modes of structures.

\section{Appendix A}

The strain components in a bar member $(k)$ can be found using the displacement field equation and the Taylor series expansion as follow:

$$
\begin{aligned}
& \varepsilon_{x x}^{(k)}=\varepsilon_{x, 0}-y^{(k)} \kappa_{y, 0}+z^{(k)} \kappa_{z, 0}+x^{(k)}\left(\frac{\partial \varepsilon_{x, 0}}{\partial x}-y^{(k)} \frac{\partial \kappa_{y, 0}}{\partial x}+z^{(k)} \frac{\partial \kappa_{z, 0}}{\partial x}\right) \\
& \varepsilon_{y y}^{(k)}=\varepsilon_{y, 0}+x^{(k)} \frac{\partial \varepsilon_{y, 0}}{\partial x}+1 / 2\left(x^{(k)}\right)^{2} \frac{\partial^{2} \varepsilon_{y, 0}}{\partial x^{2}} \\
& \varepsilon_{z z}^{(k)}=\varepsilon_{z, 0}+x^{(k)} \frac{\partial \varepsilon_{z, 0}}{\partial x}+1 / 2\left(x^{(k)}\right)^{2} \frac{\partial^{2} \varepsilon_{z, 0}}{\partial x^{2}} \\
& \varepsilon_{x y}^{(k)}=\varepsilon_{x y, 0}+\frac{1}{2} y^{(k)} \frac{\partial \varepsilon_{y, 0}}{\partial x}+\frac{1}{2} z^{(k)}\left[-\kappa_{x, 0}+\frac{\partial \varepsilon_{y z, 0}}{\partial x}\right] \\
& +x^{(k)}\left\{\left(\frac{\partial \varepsilon_{x y, 0}}{\partial x}\right)+\frac{1}{2} y^{(k)} \frac{\partial^{2} \varepsilon_{y, 0}}{\partial x^{2}}+\frac{1}{2} z^{(k)}\left[-\frac{\partial \kappa_{x, 0}}{\partial x}+\frac{\partial^{2} \varepsilon_{y z, 0}}{\partial x^{2}}\right]\right\} \\
& \varepsilon_{x z}^{(k)}=\varepsilon_{x z, 0}+\frac{1}{2} y^{(k)}\left[\kappa_{x, 0}+\frac{\partial \varepsilon_{y z, 0}}{\partial x}\right]+\frac{1}{2} z^{(k)} \frac{\partial \varepsilon_{z, 0}}{\partial x} \\
& +x^{(k)}\left\{\left(\frac{\partial \varepsilon_{x z, 0}}{\partial x}\right)+\frac{1}{2} z^{(k)} \frac{\partial^{2} \varepsilon_{z, 0}}{\partial x^{2}}+\frac{1}{2} y^{(k)}\left[\frac{\partial \kappa_{x, 0}}{\partial x}+\frac{\partial^{2} \varepsilon_{y z, 0}}{\partial x^{2}}\right]\right\} \\
& \varepsilon_{y z}^{(k)}=\varepsilon_{y z, 0}+x^{(k)}\left(\frac{\partial \varepsilon_{y z, 0}}{\partial x}\right)+\frac{1}{2}\left(x^{(k)}\right)^{2}\left(\frac{\partial^{2} \varepsilon_{y z, 0}}{\partial x^{2}}\right)
\end{aligned}
$$

Using the strain components evaluated at the centre of a bar member $(k)$ in the fundamental element, the total strain of the member can be found as [2,11-14]: 


$$
\varepsilon^{(k)}=\sum_{i=x}^{z} \sum_{j=x}^{z} \varepsilon_{i j}^{(k)} l_{i}^{(k)} l_{j}^{(k)}
$$

where, $l_{i}^{(k)}$ and $l_{j}^{(k)}$ are the directional cosines of the strain components along the bar member. Finally, the strain energy of the fundamental element made of the bar members can be found using the following formula.

$$
U=\frac{1}{2} \sum_{\text {members }} E^{(k)} A^{(k)} L^{(k)}\left(\varepsilon^{(k)}\right)^{2}
$$

\section{References}

[1] A.B. Chmielewski, Overview of Gossamer Structures, Gossamer Spacecraft: Membrane and Inflatable Structures Technology for Space Applications, edited by C.H.M. Jenkins, Vol. 191, Progress in Astronautics and Aeronautics, AIAA, Reston, Virginia, 2001 , pp. $2-3$.

[2] A. Salehian, T.M. Seigler and D.J. Inman, Dynamic Effects of a Radar Panel Mounted on a Truss Satellite, AIAA Journal 45(7) (2007), $1642-1654$.

[3] A.K. Noor, Continuum modeling for repetitive lattice structures, Applied Mechanics Review 41(7) (1988), 285-296.

[4] D.L. Dean and R.R. Avent, State of the Art of Discrete Field Analysis of Space Structures, Proceedings of the Second International Conference on Space Structures, University of Guildford, England, 1975, 7-16.

[5] C.T. Sun and S.W. Liebbe, Global-Local Approach to Solving Vibration of Large Truss Structures, AIAA Journal 28(2) (1990), $303-308$.

[6] A.H. Nayfeh and M.S. Hefzy, Continuum Modeling of Three Dimensional Rod-like Discrete Structures, Proceedings of the International Symposium on Continuum Models of Discrete Systems, University of Waterloo, Waterloo, Canada, 1977, pp. $339-357$.

[7] M.S. Lake and E.C. Kalng, Generation and Comparison of Globally Isotropic Space-Filling Truss Structures, AIAA Journal 30(5) (1992), 1416-1424.

[8] C.T. Sun and B.J. Kim, Continuum Modeling of Periodic Truss Structures, Damage Mechanics and Continuum Modeling, edited by N. Stubbs and D. Krajcinovic, American Society of Civil Engineers, New York, 1985, 57-71.

[9] U. Lee, Dynamic Continuum Plate Representations of Large Thin Lattice Structures, AIAA Journal 31(9) (1993), $1734-1736$.

[10] K. Heki and T. Saka, Stress Analysis of Lattice Plates As Anisotropic Continuum Plates, Proceedings of the 1971 IASS Pacific Symposium Part II on Tension Structures and Space Frames, Tokyo and Kyoto, Japan, 1972, pp. 663-674.

[11] A. Salehian, E.M. Cliff and D.J. Inman, Continuum Modeling of an Innovative Space Based Radar Antenna Truss, American Society of Civil Engineers Journal of Aerospace, Festschrift issue 19(4) (2006), 227-240.

[12] A. Salehian and T.M. Seigler, Dynamic Analysis of an Inflatable Rigidized Lattice Satellite Structure with Embedded Macro-fiber Composite Actuators, submitted to Shock and Vibration Journal, in review.

[13] A.K. Noor, W.H. Greene and M.S. Anderson, Continuum Models for Static and Dynamic Analysis of Repetitive Lattices, Proceedings of the AIAA/ASME/SAE 18th Structures, Structural Dynamics and Materials Conference, San Diego, California, 1977, pp. 299-310.

[14] A.K. Noor, M.S. Anderson and W.H. Greene, Continuum Models for Beam and Plate-like Lattice Structures, AIAA Journal 16(12) (1978), 1219-1228.

[15] A.K. Noor and C.M. Andersen, Analysis of Beamlike Lattice Trusses, Computer Methods in Applied Mechanics and Engineering 20(1) (1979), 53-70.

[16] A.K. Noor and W.C. Russell, Anisotropic Continuum Models for Beamlike Lattice Trusses, Computer Methods in Applied Mechanics and Engineering 57(3) (1986), 257-277.

[17] A. Salehian and D.J. Inman, Dynamic Analysis of a Lattice Structure by Homogenization: Experimental Validation, Journal of Sound and Vibration 316(1-5) (2008), 180-197.

[18] A. Salehian and D.J. Inman, Micropolar Continuous Modeling and Frequency Response Validation of a Lattice Structure, ASME Journal of Vibration and Acoustics 132(1) (2010), 1-7. 

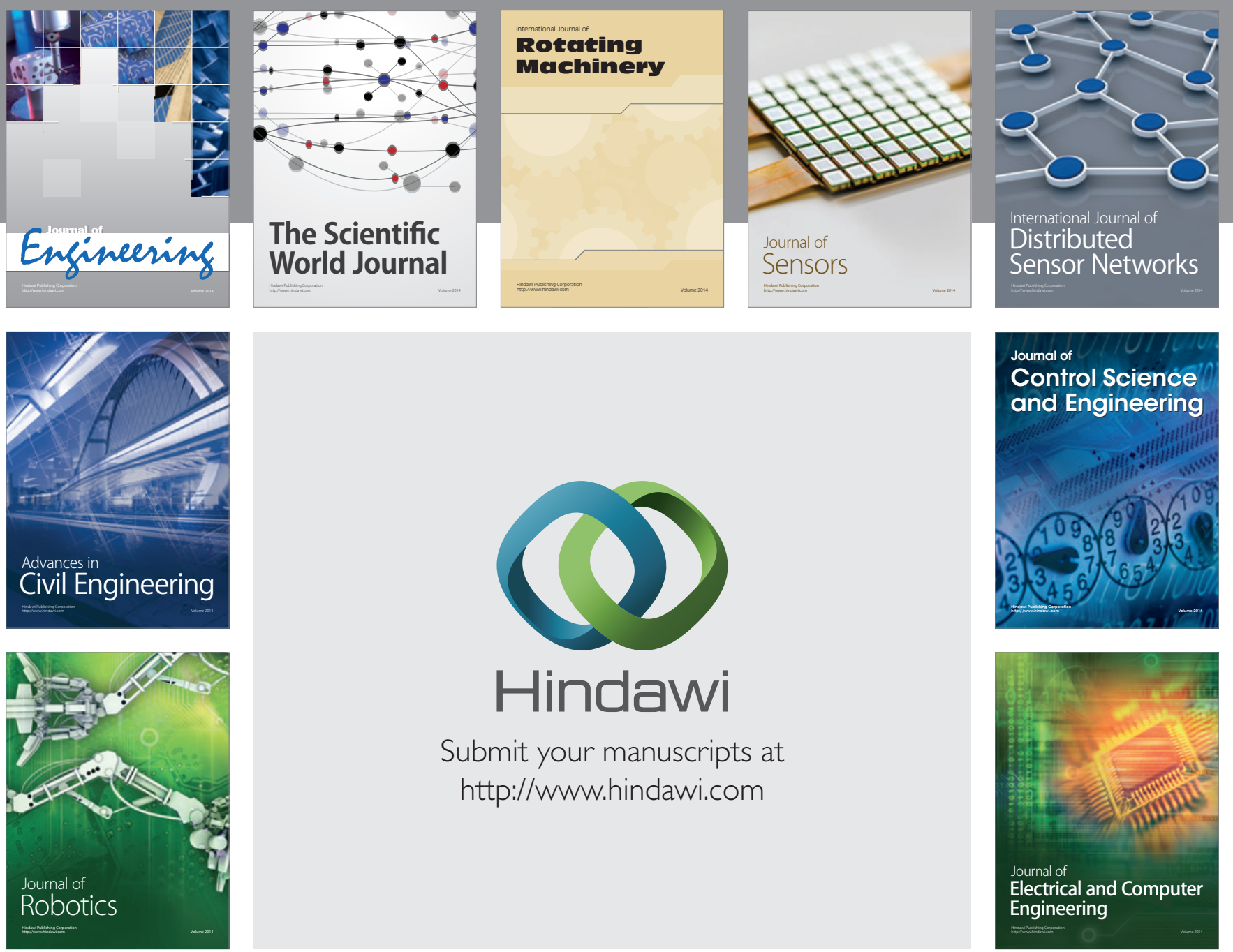

Submit your manuscripts at

http://www.hindawi.com
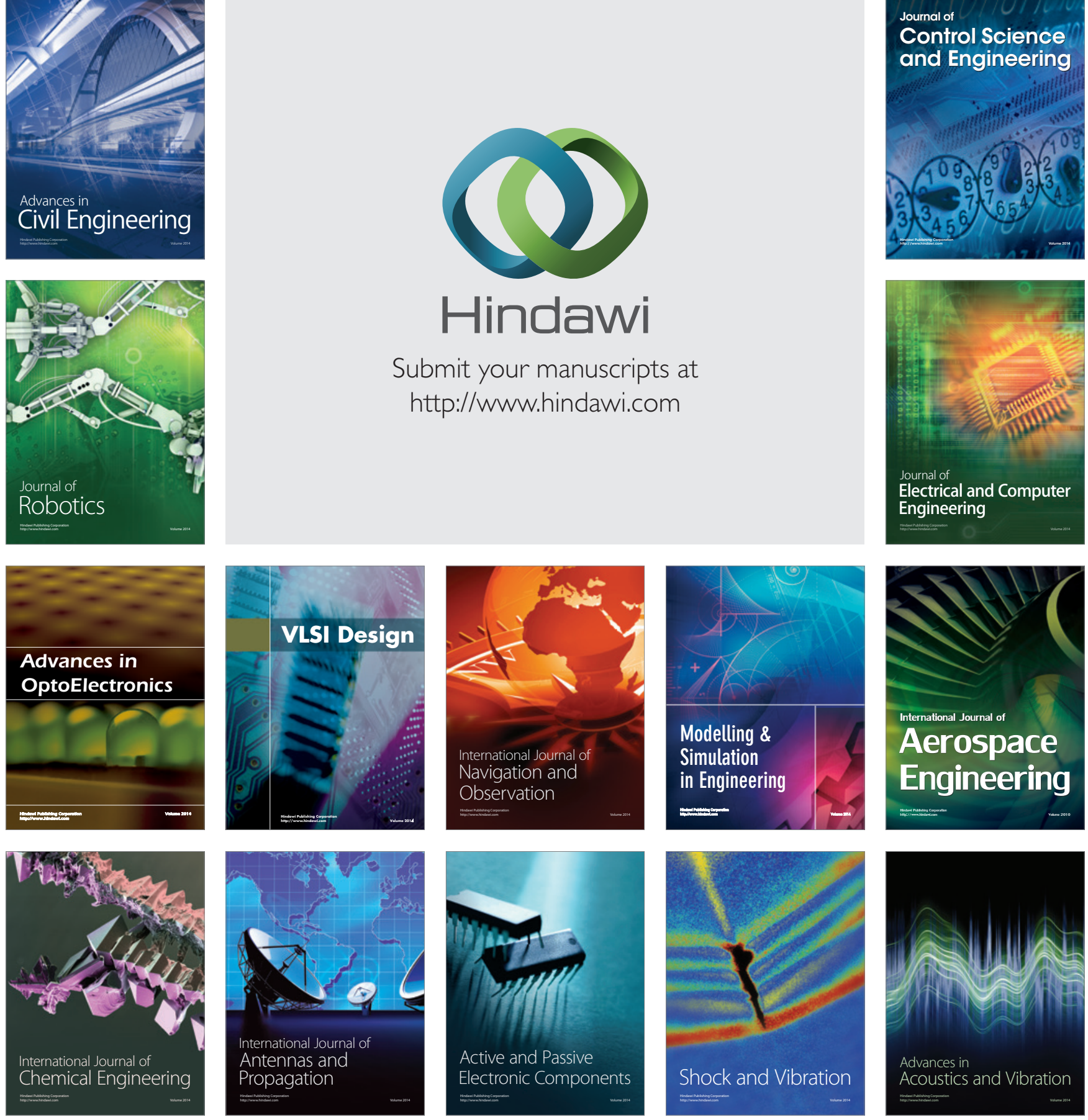